\title{
Coupling effects in QD dimers at sub-nanometer interparticle distance
}

\author{
Carlo Nazareno Dibenedetto ${ }^{1,2,}$, Elisabetta Fanizza ${ }^{1,2,}$, Rosaria Brescia ${ }^{3}$, Yuval Kolodny ${ }^{4}$, Sergei Remennik ${ }^{4}$, \\ Annamaria Panniello ${ }^{1}$, Nicoletta Depalo ${ }^{1}$, Shira Yochelis ${ }^{4}$, Roberto Comparelli ${ }^{1}$, Angela Agostiano ${ }^{1,2}$, Maria Lucia \\ Curri $^{1,2}$, Yossi Paltiel ${ }^{4}$, and Marinella Striccoli ${ }^{1}(\triangle)$ \\ ${ }^{1}$ CNR-Istituto per i Processi Chimico-Fisici SS Bari, Via Orabona 4, 70125 - Bari, Italy \\ ${ }^{2}$ Dipartimento di Chimica, Università degli Studi di Bari Aldo Moro, Via Orabona 4, 70125-Bari, Italy \\ ${ }^{3}$ Electron Microscopy Facility, Istituto Italiano di Tecnologia, via Morego 30, 16163 Genova, Italy \\ ${ }^{4}$ Department of Applied Physics and the Center for Nanoscience and Nanotechnology, Hebrew University of Jerusalem, Jerusalem 9190401, Israel \\ ${ }^{\S}$ Carlo Nazareno Dibenedetto and Elisabetta Fanizza contributed equally to the work.
}

(C) The Author(s) 2020 .

Received: 16 December 2019 / Revised: 5 March 2020 / Accepted: 6 March 2020

\begin{abstract}
Currently, intensive research efforts focus on the fabrication of meso-structures of assembled colloidal quantum dots (QDs) with original optical and electronic properties. Such collective features originate from the QDs coupling, depending on the number of connected units and their distance. However, the development of general methodologies to assemble colloidal QD with precise stoichiometry and particle-particle spacing remains a key challenge. Here, we demonstrate that dimers of CdSe QDs, stable in solution, can be obtained by engineering QD surface chemistry, reducing the surface steric hindrance and favoring the link between two QDs. The connection is made by using alkyl dithiols as bifunctional linkers and different chain lengths are used to tune the interparticle distance from few $\mathrm{nm}$ down to $0.5 \mathrm{~nm}$. The spectroscopic investigation highlights that coupling phenomena between the QDs in dimers are strongly dependent on the interparticle distance and QD size, ultimately affecting the exciton dissociation efficiency.
\end{abstract}

KEYWORDS

quantum dots, dimers, surface chemistry, dithiols, coupling

\section{Introduction}

Research on nanomaterials and their technological application is rapidly increasing in the last few years. Currently, colloidal semiconductor quantum dots (QDs) are used for application in a plethora of photonic [1] and optoelectronic [2,3] devices, ranging from commercial TV displays [4] to LEDs [5], solar cells [6, 7], miniaturized sensors [8], biochips [9], etc. Most of such optoelectronic and sensing applications require an efficient coupling among the QDs deposited in solid disordered aggregates, with limited success. The colloidal QDs are typically surrounded by an insulating shell of capping molecules, such as long alkyl chain phosphine, phosphine oxide, amine, fatty acid. The organic ligands are essential to control the nucleation and growth of the QDs during the synthesis and are responsible for their electro-neutrality and steric stabilization in solution, keeping separate individual QDs and preventing aggregation and mutual coupling. The potential barrier created by the ligand shell results in a negligible overlap of the electronic wavefunctions across the QDs [10]. Several treatments aiming at engineering the QD surface functionalization have been proposed in order to promote an effective interaction between the QDs [11]. However, the electronic structure, and thus the optical properties of the QDs, strongly depends on the surface status, including type of ligands and their interaction with the QD surface. Therefore, any chemical treatment of the QD surface profoundly influences their electronic structure.

Numerous reports have demonstrated the dramatic effect of surface modification in reducing interdot separation on electronic coupling and/or energy transfer rate [11-13]. Paltiel et al. [14] has described the fabrication of a disordered layered QDs structure, coupled with alkyldithiols. The exchange of the native QD insulating capping ligands has been performed directly on deposited films of QDs with thiols with shorter alkyl chain lengths. Also, Talapin and Murray [15] has shown that treatment with hydrazine of PbSe QD films allows to remove the pristine oleic acid ligand coordinating QD surface and to decrease the interdot spacing from 1.1 to $0.3 \mathrm{~nm}$, that reflects an increase of the conductivity through the QD array by ten orders of magnitude.

These studies generally describe the fabrication of disordered solid films based on QDs and their coupling properties. However, to reduce competing mechanisms and collective effects due to large aggregates of QDs, a controlled number of interacting particles in solution is amenable. Indeed, investigation in solution of such QD based assemblies, dimers or oligomers, may offer a more comprehensive understanding of the mechanisms underlying the coupling. 
Colloidal nanoparticles (NPs) in solution behave as quasistable systems and, under certain conditions, spontaneously undergo bulk aggregation [16]. Hence, the formation of small assemblies of NPs may be achieved through a delicate balance between repulsive interactions, responsible for colloidal stability, and attractive forces leading to aggregation. In recent literature $[17,18]$, dimers of colloidal NPs have been prepared mainly by using two types of interactions, namely electrostatic interaction, generally for metallic NPs [17], and molecular bridging (as DNA, organic molecules, etc.) to connect the two colloidal units [18]. It is worth to underline that experimental methods available in both cases are rather specific and, in some cases, limited by low yield, low stability, and poor reproducibility and scalability. Therefore, possible applications of these systems are scarce, thus remaining mainly subject of fundamental studies and proof-of-concept experiments. A successful electrostatically driven dimerization approach relies on the careful adjustment of relevant parameters as ionic strength, dielectric constant and particle size to finely tune the entity of the repulsion forces, and thus address the conditions that favour controlled self-assembly [19]. As an example, direct self-assembly of citrate-capped $\mathrm{Au}$ NPs in solution have been obtained in the presence of $\mathrm{NaCl}$ [20] and the extent of the aggregation could be roughly controlled shielding the electrostatic repulsion between the NPs by suitably increasing the ionic strength. Although the dimers can be obtained with high purity after gradient centrifugation, the initial yield in dimer is low, due to the limited control on their formation. Recognition mechanisms of biological molecules (DNA, antidig-antibody etc.) were also exploited for the fabrication of dimers of colloidal NPs, as widely reported [21-23]. However, such an approach is not suited to obtain interparticle distance shorter than few $\mathrm{nm}$, due to the nature of the used molecules [24].

The presence of the organic shell surrounding the NPs can be also exploited, taking advantage of the relatively simple procedures of capping exchange and the large variety of available functionalization agents to use as bifunctional linker to connect the units and, thus, obtain NP dimers and oligomers [25]. Starting from NPs in solution, assembling of NPs in dimers by means of a bifunctional molecule can be seen as a stepgrowth reaction. The yield of dimer formation is directly related to the ability of such bifunctional molecule to bind NP surface that is dictated by the specificity of the local interaction. The possibility that a bifunctional linker may connect two NPs requires a properly engineered NP surface chemistry with suitable surface ligands. In addition, the dynamic equilibrium of ligands in solution and at the NP surface needs to be taken in due account. Finally, bifunctional linkers offer the remarkable advantage to control the interparticle distance.

The fabrication of dimers based on II-VI semiconductor QDs in solution and the investigation of their properties are still at their infancy, due to the small sizes of the QDs and the complexity of the procedures of surface functionalization. Peng et al. [26] have demonstrated the first example of dimer fabrication by ligand cross-linking. Koole et al. [27] have studied the degree of electronic coupling as a function of the size of the QDs in stable dispersions of molecular-like aggregates of CdTe QDs cross-linked with 1,6-hexanedithiol, supporting the study with quantum mechanical calculations. The best coupling has been demonstrated with small QDs, due to larger extension of the wavefunction outside the QD. Basche et al. [28] have investigated the QD dimers and trimers assemblies in solution by either direct coupling of particles via ligand depletion or by a particles cross-linking realized using a rigid bifunctional dye molecule as a bridge.
Here, the design, the fabrication in solution and the optical and morphological characterization of nanostructured dimers based on CdSe QDs, with control on interparticle distance, are reported. The general strategy relies on a two-step procedure: firstly, a treatment with short alkyl chain amines aims at partially replacing the native ligands to reduce their steric hindrance at the QD surface, and, then, a functionalization procedure with alkyl dithiols acting as bifunctional linkers promotes the formation of QD dimers. The -SH head groups bind the surface of the two QDs in the dimeric structure while the alkyl chain defines the interparticle distance. Alkyl dithiols with different alkyl chain length (i.e., 1,3-propanedithiol (pDT), 1,6-hexanedithiol (hDT), 1,8-octanedithiol (oDT) and 1,16-hexadecanedithiol (hdDT)) have been investigated to study the dependence of the coupling phenomena on the interparticle distance. To control the stoichiometry of the final QD-based assemblies, achieve maximal dimer yield (DY), and avoid aggregates formation, a delicate balance between QDs and bifunctional ligands concentration is required. The dimer formation process, monitored by transmission electron microscopy (TEM), Ultraviolet visible (UV-Vis) absorbance spectroscopy, stationary and time resolved fluorescence spectroscopy, has been elucidated and the effect of the interparticle distance on their properties investigated.

\section{Results and discussion}

\subsection{Dimers fabrication and characterization}

The dimeric structures are fabricated by using bifunctional alkyl ligands with an A-Y-A structure, able to directly replace the pristine ligands at the CdSe QDs surface and connect the two QDs by means of the "A" head group, being "Y" the alkyl chain acting as a spacer to define the interparticle distance. For this purpose, the bifunctional ligand must be dispersible in the same solvent of the QDs and the A group must possess a good affinity to the QDs surface. Under these conditions, an effective binding of the linker is expected, essential for the formation of a "robust" dimeric system, with controlled interparticle distance.

Bifunctional ligands, namely 1,3-propanedithiol (pDT), 1,6-hexanedithiol (hDT), 1,8-octanedithiol (oDT) and 1,16hexadecanedithiol (hdDT), with a nominal length, according to the Tanford's formula [29], of $0.55,0.9,1.3$, and $2.2 \mathrm{~nm}$, respectively, are selected as molecular linkers for the dimer preparation. These molecules are highly soluble in hexane, the weekly polar organic solvent dispersing the QDs, and have a thiol as A head group easily binding the QD surface, due to its high affinity to CdSe surface [30], and Y an alkyl chain formed of three (C3), six (C6), eight (C8) and sixteen (C16) methylene groups, respectively. The use of a series of alkyldithiol homologues enables the investigation on the effect of the alkyl chain length on coupling.

Colloidal CdSe QDs of two different sizes, 4,4 nm (QD1) and 3,7 nm (QD2) diameter, respectively, have been purposely prepared to investigate the effect of the NP dimension on dimer formation and coupling phenomena, as QD size affects both curvature radius, and hence surface ligand packing and exchange reaction, and the wavefunction extension outside the QD. To maximize the delocalization and minimize the core distance, bare CdSe QDs have been investigated, rather than the most popular CdSe@ZnS core-shell nanocrystals. The synthesis is carried out by hot injection approach (see Experimental Section for details), using for the preparation of each sample the same coordinating solvent reaction mixture made of trioctylphosphine oxide (TOPO), hexadecylamine (HDA) and tributylphosphine (TBP), the same precursor concentration 
and injection temperature and selecting two different reaction time, namely $180 \mathrm{~s}$ for QD1 and $90 \mathrm{~s}$ for QD2, respectively. The longer the reaction time the larger the QD diameter is, and the more red shifted their spectroscopic absorption and emission features are. The morphologic analysis of both samples, reported in Fig. 1, indicate $4.4 \mathrm{~nm}(\sigma \%=7 \%$, Fig. 1(a)) as average diameter (d) of QD1 and $3.7 \mathrm{~nm}(\sigma \%=8 \%$, Fig. 1(b)) for QD2. The spectra of the QD1 dispersion in hexane are characterized by a first excitonic transition centered at $617 \mathrm{~nm}$ and an intense emission peak at $625 \mathrm{~nm}$ (Fig. 1(c)), while QD2 sample, prepared at shorter reaction time, has a blue shifted first excitonic transition centered at $590 \mathrm{~nm}$ with a PL band at $600 \mathrm{~nm}$ (Fig. 1(c)). The low standard deviation value $(\sigma \%)$, the narrow photoluminescence signal (full width half maximum of about $80 \mathrm{meV}$ ) and the absorption line profile, that clearly shows first and high order energy excitonic transitions, all confirm a narrow dispersion in sizes of the two samples. The QDs are characterized by an absolute PL QY value of $24.1 \%$ for QD1 and 24.6\% for QD2 and the recombination dynamics is not significantly affected by the size (Fig. S1 in the Electronic Supplementary Material (ESM)).

Both the synthesized CdSe QDs samples present the same ligand shell coordinating their surface and composed of oleate, TOPO, HDA and TBP [31]. According to ligand classification described by Anderson et al. [32] oleate is an X type ligand, while TOPO, HDA and TBP are L type ligands. The X type ligands are generally neutral radicals binding neutral surface sites (each with an unpaired electron) or, more commonly, monovalent ions binding oppositely charged sites at the NC surface, while L type ligands are neutral two-electron donors with a lone electron pair coordinating surface metal atoms by dative covalent bonds. Ligands are required to ensure the electroneutrality of the QDs, therefore, any ligand exchange reaction at QDs surface, replacing the pristine ligand with new molecules, should preserve such a condition.

The strategy to obtain dimers of QDs may follow two possible routes, either a single step, direct functionalization of the QDs with the bifunctional linker (Scheme 1(a)) or a two-step approach, characterized by a preliminary surface treatment prior to the bifunctional linker functionalization (Scheme 1(b)). Both routes, to induce dimer formation, require alkyldithiol bifunctional linkers in excess to be added to a dilute solution of the as prepared QDs in hexane.

In general, several factors such as solvent polarity, ligand
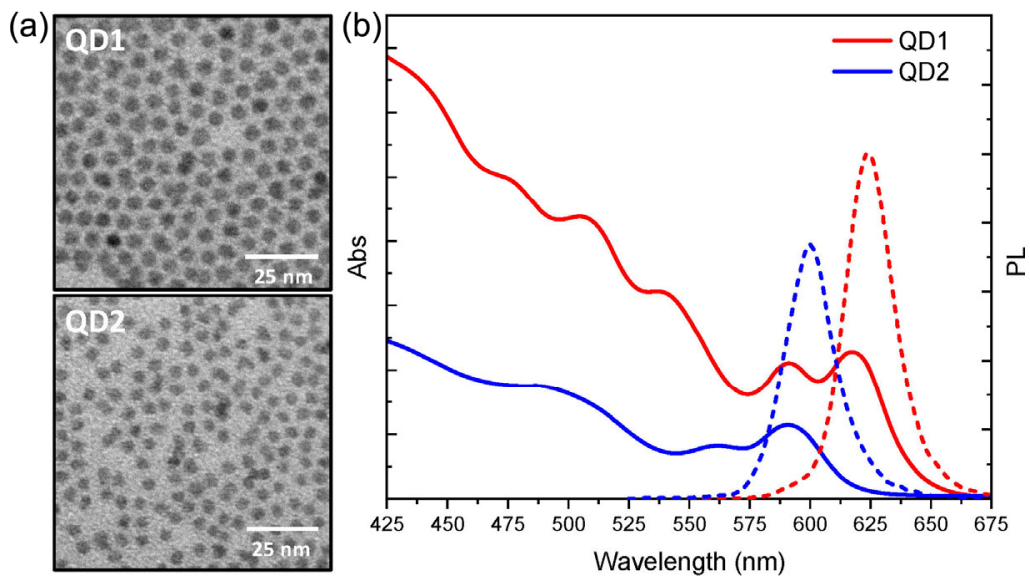

Figure 1 (a) TEM micrograph and (b) absorption and emission spectra of QD1 $(d=4.4 \mathrm{~nm})$ and QD2 $(d=3.7 \mathrm{~nm})$ in hexane solution $\left(\lambda_{\text {exc }}=485 \mathrm{~nm}\right)$.

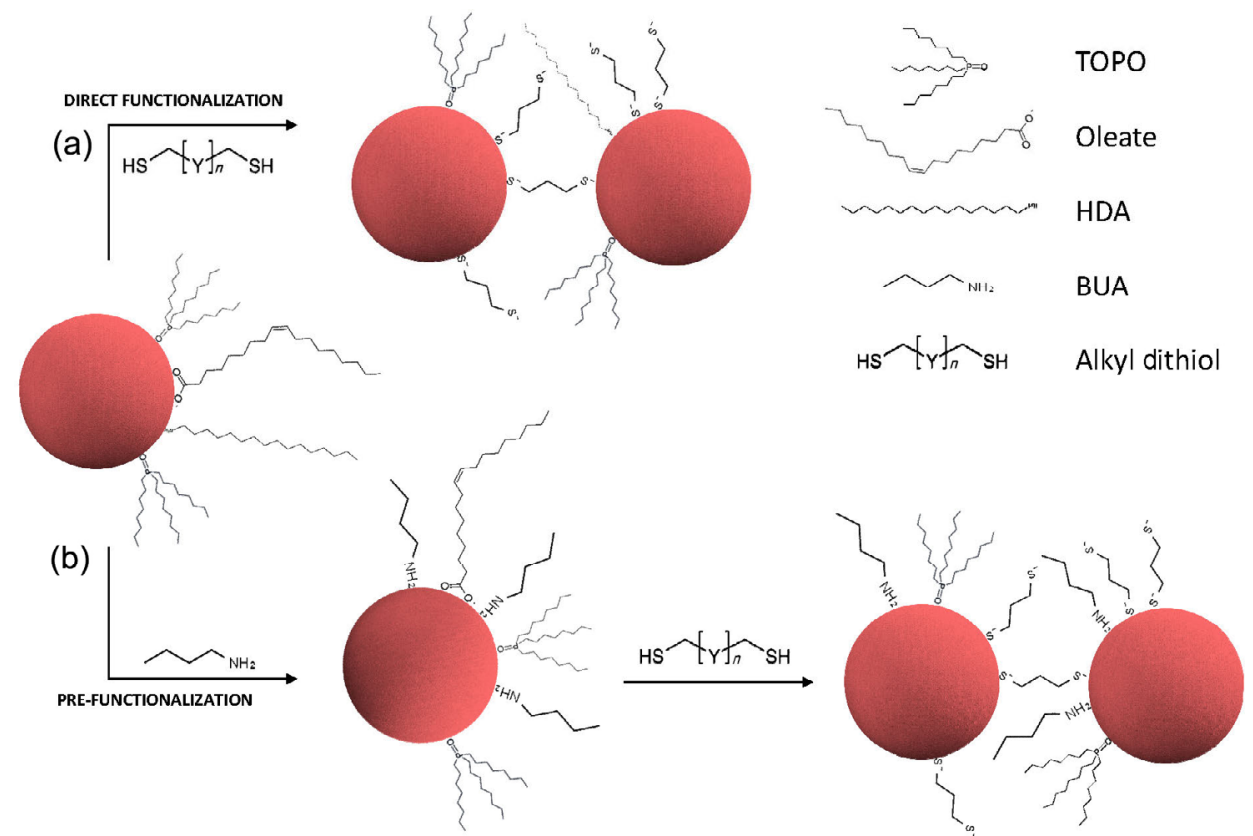

Scheme 1 Functionalization strategies for dimer formation. (a) Dimer formation by direct ligand exchange at the QD surface with an alkyl dithiol and (b) the two step functionalization approach based on a preliminary treatment with a short alkyl chain amine and the subsequent ligand exchange with the alskyl dithiol (figure is not drawn to scale). 
headgroup affinity for the QD surface and relative abundance of both incoming and leaving ligands affect the kinetics and mechanism of ligand exchange. Here, mass action and the high affinity of thiol head-group are expected to favor the displacement of the pristine ligands and the binding at the $\mathrm{QD}$ surface, while the low concentration of the QD dispersion should limit their aggregation. The chemistry behind the exchange of the pristine ligands with alkyl thiols and their binding at CdSe QDs surface has been largely debated [33] and different mechanisms have been proposed to describe the thiol exchange reaction. Thiol has been established to binds as thiolate the QD surface, behaving as an $\mathrm{X}$ type ligand [32]. Therefore, in the case of the oleate, TOPO, HDA and TBP capped CdSe NCs, the thiol is anticipated to replace oleate, in order to preserve the QD electro-neutrality. Indeed, exchange of such tightly bound $\mathrm{X}$ type ligands probably takes place in nonpolar solvent by an associative pathway [34]. However, the low binding energy of the L type ligands (HDA, TOPO, TBP) that are, in fact, expected to rapidly adsorb and desorb at the NC surface, even at room temperature, does not allow to completely rule out additional mechanisms, mainly based on L type ligand detachment and replacement [35].

The kinetics and yield of dimer formation also depend on the steric availability of the QD surface, as a sterically crowded capping layer hinders the QD surface, hampering the binding of the incoming ligands that diffuse from the solution. TOPO and HDA, characterized by long alkyl chains, are highly sterically hindered, thus likely limiting the access of the new ligand to the NC surface, especially in the case of large QDs, having low curvature radius [36]. Therefore, dimer formation is favored by increasing the amount of accessible anchor points suited for the exchange with dithiols at the NC surface. In this regard, a two steps approach is here proposed. The procedure is based on the partial replacement of the pristine $\mathrm{L}$ type ligands TOPO and HDA with an intermediate ligand, having a very short alkyl chain and a binding affinity to the QD surface lower than those characterizing thiols (Scheme 1(b)). Such a preliminary treatment, prior to the functionalization with the bifunctional linker, is expected to induce local inhomogeneity in the organic capping layer at the QDs surface that can favor the dithiol binding. Butylamine (BUA) has been selected for this purpose, being an L type ligand, highly dispersible in the QDs solvent, with a functional amine group easily binding the QD surface, even though more weakly than thiol. Then, BUA preserves the electro-neutrality of the QD, and, at the same time, it can effectively reduce the steric hindrance at QD surface, due to its short alkyl chain $\mathrm{C} 4$ and can be easily replaced by thiols.

Figure 2(a) shows the UV-Vis absorption and PL spectra of the QDs before and after the treatment with BUA, by using a BUA: QD molar ratio 500:1. This pre-functionalization step does not significantly affect the optical properties of the QDs, except for a faint enhancement in PL emission, attributed to an improved surface passivation likely induced by the short chain amine [37].

In Fig. S2 in the ESM, the TEM micrographs of QD1, treated with BUA at two different BUA:QD1 molar ratio (500:1 in Fig. S2(a) in the ESM and 20,000:1 in Fig. S2(b) in the ESM respectively) are reported. The images highlight that, while at the low BUA:QD1 molar ratio the QDs appears well separated on the TEM grid, a significant aggregation of QDs is observed at the high molar ratio.

Such an occurrence can be explained considering that, at low molar ratio, BUA is able to only partially replace the pristine ligands thus preserving the QD steric stabilization, guaranteed by the residual TOPO/HDA capping layer at the QD surface (sketch in Fig. S2(a) in the ESM). Conversely, the addition of a large amount of BUA results in a massive exchange of the pristine ligands with the short alkyl chain (C4) thiols that, consequently, brings the QDs closer to each other, and thus favor their aggregation (Fig. S2(b) in the ESM).

After such a pre-treatment with BUA, the functionalization of QD surface with the dithiol linkers is meant to lead to the formation of dimers, though, since the functionalization process is based on a statistical approach, the formation of assemblies with a number of QDs larger than two cannot be excluded. The results concerning the dimerization of QDs by using the shortest bifunctional linker of the investigated series, namely the propanedithiol (pDT), are described in Figs. 2 and 3.

A preliminary experiment has been set up by adding to the QD solution $\left(5 \times 10^{-7} \mathrm{M}\right) \mathrm{pDT}$ in hexane $\left(1.7 \times 10^{-4} \mathrm{M}\right)$ in order to estimate the $\mathrm{pDT}: \mathrm{QD}$ molar ratio suitable to maximize the DY, and still able to limit the NP aggregation. Dimer formation has been monitored in situ by UV-Vis absorbance spectroscopy (Fig. 2(b)), without further sample dilution. Since dimers are small assemblies of QDs, their formation may induce scattering in solution, as evidenced in the absorption spectra. According to the Rayleigh formulation, scattering is more pronounced at the high-energy side of the absorbance spectra, being, however, also present at low energy. A magnification of the absorption tails, in a spectral region where no electronic transitions are expected, is shown in the inset of Fig. 2(b). The dimer formation can be monitored looking at the extent of the Rayleigh scattering, while adding increasing amount of pDT, thus identifying the $\mathrm{pDT}$ :QD molar ratio value corresponding to the maximum extent of the small assemblies formation, just before major aggregation phenomena take place. The increment
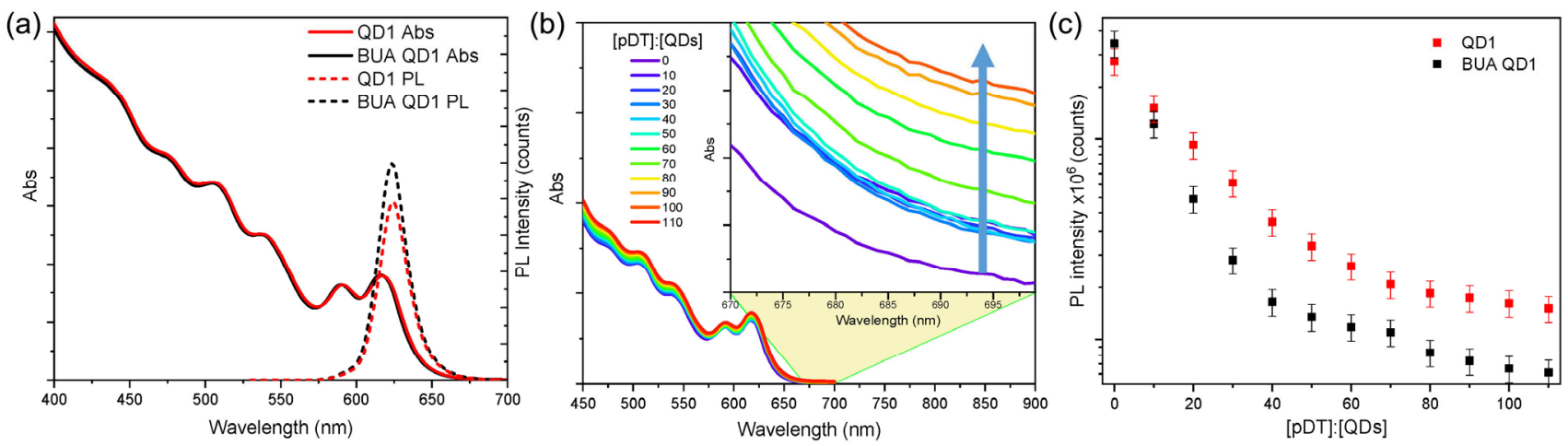

Figure 2 (a) UV-Vis absorption (solid line) and PL (dotted line) spectra of QD1 before (red line) and after (black line) the treatment with BUA. (b) UV-Vis absorption spectra of QD1 in hexane solution at different molar ratio [pDT]/[QDs]. In the inset, a magnification of the red tail of the absorption spectra. The arrow indicates increasing molar ratio [pDT]/[QDs]. (c) PL intensity of QDs in solution with pristine capping (red points) and treated with BUA (black points). 
of pDT in the QDs solution induces an increase in the scattering that is negligible in the initial phase and remains constant for pDT:QD molar ratio between 10:1 and 50:1. Subsequently, any additional increase in the $\mathrm{pDT}$ :QD molar ratio leads to a remarkable increase in scattering contribution, which is a clear evidence of a the higher and higher extent of QD aggregation. The spectroscopic analysis has allowed to identify 50:1 as the highest $\mathrm{pDT}$ :QD molar ratio suitable to obtain dimers with negligible formation of larger QD aggregates.

The distinctive role played by the BUA in the formation of dimers can be clearly inferred by comparing the PL intensity at maximum emission wavelength of the native and of the BUA pre-treated (BUA:QD 500:1) QD1 sample at increasing pDT:QD molar ratio (Fig. 2(c)). As expected, thiols induce a quenching of the PL, due to the formation of hole trap states, and the higher the amount of thiols binding at the QD surface is, the more pronounced the PL quenching is expected. Therefore, since the quenching measured for BUA-treated QDs is stronger than that detected for the as prepared QD, when treated at the same $\mathrm{PDT}$ :QD molar ratio, the pre-treatment with BUA can be considered responsible for a larger amount of dithiol binding the surface of the QDs. The data of Fig. 2(c), for pDT:QD molar ratio below 50:1, prior of the aggregation, allow to evaluate the effect of the thiols on the spectroscopic properties of the dimers.

The dimer formation has been assessed by means of TEM images of the BUA treated QD1 after functionalization with pDT (Figs. 3(a)-3(d), see also Fig. S3 in the ESM).

It is important to emphasize that solvent evaporation, occurring during TEM grid preparation, may induce possible assemblies of QDs that, though may look like dimers in the micrograph, are, instead, aggregates resulting from van der Waals interactions among the hydrophobic chains of the QDs. Therefore, to limit such possible artefacts, the samples have been further diluted (1:60) before the deposition on the TEM grid [38]. The TEM images (Figs. 3(a), and Figs. S3 and S6 in the ESM) provide a clear and decisive evidence of dimer formation (see Experimental Section), with a negligible aggregation. The investigation, performed by using a high angle annular dark field (HAADF) equipped on probe corrected scanning transmission electron microscope (STEM), has allowed us to measure an interparticle distance between the QDs in the dimeric structures (Figs. 3(b) and 3(c)) of a few tenths of a nm (Fig. S4 in the ESM), essentially in agreement with the geometrical distance defined by the length of the $\mathrm{C} 3$ chain of the pDT $(0.55 \mathrm{~nm})$. However, due to the $2 \mathrm{D}$ character of the TEM images and to dependence of the measurement on the QD orientation, the value determined from the STEM micrographs is affected by some uncertainty and should be considered on the estimation of the QD distance.

Similar results have been attained for QD2 sample (Figs. 3(c) and 3(d)) and by using as linker the alkyldithiol homologues (hDT, oDT, hdDT) at the same molar ratio of $50: 1$, thus confirming the reproducibility of the approach used for fabrication of dimeric assemblies of QDs of different sizes (Fig. S5 in the ESM).

The effectiveness of the preliminary treatment with BUA in the formation of dimers is confirmed by comparing the morphological analysis of the as prepared and BUA pre-treated QD1 sample at increasing pDT:QD molar ratio (Fig. S6 in the ESM). When the bifunctional linker is exchanged at the surface of the as prepared QDs at pDT:QD = 50:1, a lower dimer yield (DY) is achieved (Fig. S6(a) in the ESM), while significantly higher values $(>60 \%)$ are found for the exchange performed on the BUA pre-treated sample (Fig. S6(b) in the ESM). Further addition of pDT induces aggregation phenomena in both samples (Figs. S6(c) and S6(d) in the ESM). Density gradient ultracentrifugation procedure has been performed to further improve the DY. The QD dimers in hexane solution were chemically and optically stable in time for few months.

A comprehensive spectroscopic study, by steady state and time resolved, fluorescence has been performed to investigate the QD dimer formation (Fig. 4, and Fig. S7 in the ESM) and evaluate the occurrence of coupling effects therein. While the thiol functionalization is typically known to induce quenching of the CdSe QDs emission, due to the trapping of the photogenerated holes caused by thiol group binding to the QD surface [39], the mutual QD proximity in the dimers and the consequent overlap of their wavefunctions may further affect the emission properties. To discriminate this latter effect from that due to the binding of thiol to the QD surface on PL features, and also to determine the extent of the PL quenching due to the thiol groups, a comparison between the QD1 samples treated with alkyl thiol and dithiol homologues, respectively, at the same molar ratio, has been performed. Thiols having the same alkyl chain length $(0.55$ and $1.3 \mathrm{~nm}$, respectively for the $\mathrm{C} 3$ and the C8 molecules) have been selected for this purpose (see also Fig. S7 in the ESM for QD2).

Such a comparison is expected to provide insights on possible exciton dissociative mechanisms, such as energy transfer between the QDs, electronic coupling, charge transfer from QD to the surface ligand or surface charge trapping induced by the thiol group. In case of an energy or charge transfer from QDs to the ligand or of a surface trapping state due to the thiol group binding the QD surface, the exciton lifetime is expected to strongly decrease for both thiols and dithiols functionalized QDs.

Figure 4(a) reports the intensity of the PL band maximum for the BUA treated QD1 samples before and after the functionalization with each alkyl thiols and dithiols in the investigated homologue series (oDT, oT, hDT, pDT, pT). A significant quenching of the luminescence in presence of both
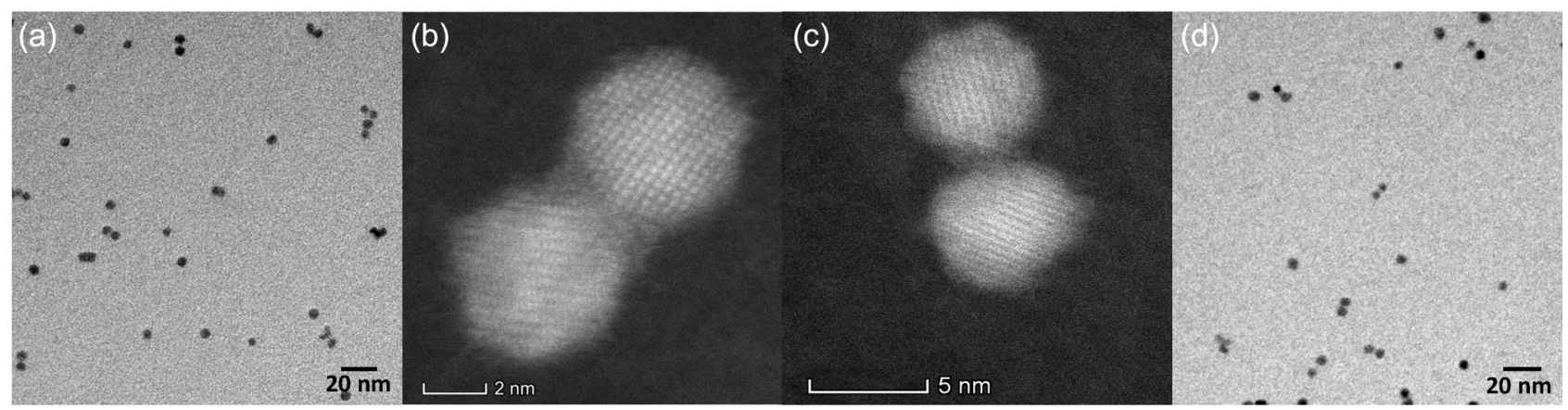

Figure 3 TEM micrographs ((a) and (d)) and HAADF STEM ((b) and (c)) micrographs of QD1 (panel (a) and (b)) and QD2 (panel (c) and (d)) dimers, prepared with pDT. 

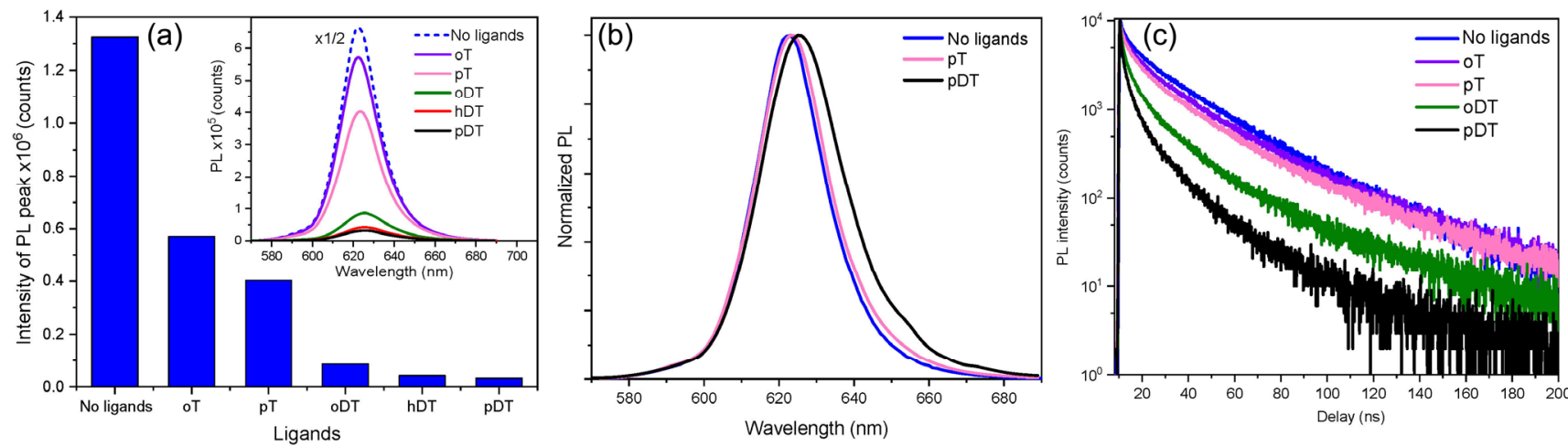

Figure 4 (a) Bar plot of the emission intensity of BUA-treated QD1 samples before and after functionalization with thiols and dithiols homologues at the DT: QD 50:1 molar ratio. In the inset, the corresponding PL spectra. (b) Normalized PL spectra of BUA treated QD1 (blue line), after functionalization with the pT (pink line) and pDT (black line). (c) Decay profiles of BUA treated QD1 sample (blue line) and after addition of oT (violet line), oDT (green line), pT (pink line) and pDT (black line) $\left(\lambda_{\text {exc }}=485 \mathrm{~nm}\right)$.

thiols and dithiols is evident from the bar plot (Fig. 4(a)). However, PL quenching is stronger for dithiols with respect to thiols and gets even stronger when the alkyl chain length reduces from $\mathrm{C} 8$ to $\mathrm{C} 3$ in the homologue series. In case of thiols (oT, $\mathrm{pT}$ ), such a dependence of the quenching extent from the chain length cannot be explained straightaway, since the experiments have been performed at the same [Thiol]:[QDs] ratio, and, therefore, in principle, the same amount of $\mathrm{SH}$ groups is available to bind to QDs, regardless of the chain length of the thiol. However, considering that the shorter chains of $\mathrm{pT}$ molecules are more likely to enter the organic shell coordinating the QDs, $\mathrm{pT}$ is likely to interact more effectively than oT molecules with the QD surface. As further evidenced, the normalized PL spectra (Fig. 4(b)) of QD solutions functionalized with pDT have a more pronounced red shift, with respect to the small value measured for thiols. Such a difference in PL shift cannot be only attributed to an electronic reconfiguration of the energy bands after thiol binding, since the - $\mathrm{SH}$ group is present in both pT and pDT molecules and the distribution in size of QDs is the same. In addition, the PL decays (Fig. 4(c)) show that the profile for thiols treated QDs almost overlaps the decay of the BUA treated QDs, without any significant influence of the length of the chain. A very different behavior is observed for QDs functionalized with dithiols (both $\mathrm{pDT}$ and $\mathrm{oDT}$ ) that show a faster decay with respect of BUA treated QDs and dependent on the dithiol alkyl chain, being more rapid as the alkyl chain get shorter, and thus for smaller interparticle distance. In fact, dithiols with diverse chain length are not expected to shift differently the energy levels of QD, rather only to modify the width of the potential barrier, due to the localized nature of the $\sigma$-orbitals on the alkyl chains. In addition, the variation in PL lifetime cannot be attributed to a change in dielectric environment, which is similar both for thiols and dithiols, also due to the very limited amount of ligand molecules added to the QD dispersion.

This evidence highlights that additional phenomena take place upon dithiol capping exchange of the QDs and their assembly in dimeric structures. It is worth noticing that, even after purification procedures, few isolated QDs and some larger QD aggregates may still be dispersed in solution, thus contributing to the PL steady state and time resolved signals.

The PL decay profiles (Fig. 4(c) and Fig. S1 in the ESM) have been best fitted by a three or four exponential function and the average decay times, calculated as indicated in the ESM, are reported in Table 1 for both QD1 and QD2 samples in hexane solution.

The obtained average decay times of $\mathrm{pT}$ and oT treated QDs are comparable to the lifetime of both sized BUA treated QDs, while the functionalization with dithiols as bifunctional linkers and the consequent dimer formation results in a faster decay, down to few ns for $\mathrm{pDT}$, and even lower for the small QD2. This additional reduction in exciton recombination time cannot be ascribed only to thiol surface trapping phenomena, as evidenced from the decays of QDs functionalized with thiols, but is rather an evidence of inter-dots coupling affecting the exciton recombination dynamics. Conversely, the short interparticle distance seems to play a major role. Indeed, the red shift of the steady state emission and the faster PL decay times of the dithiol linked QDs in the dimers prove that transfer or coupling phenomena may only take place between QDs close enough and connected.

\subsection{Coupling of QDs}

In order to understand the coupling between QDs and to identify whether energy or charge transfer phenomena may take place between the dimer components, some hypotheses can be done. Let us first consider that a Forster resonance energy transfer (FRET) may take place between the two QDs in a dimer. FRET process is a non-radiative energy transfer between a fluorescent donor and a lower energy acceptor via a long range dipole-dipole interaction. In most reports $[40,41]$ investigating FRET processes between two organic fluorophores [40, 41] or between dyes and QDs [41], FRET is demonstrated by a decrease in the donor emission intensity with the concomitant increase of the acceptor emission, and by a decrease in donor lifetime associated with an increase in acceptor lifetime, due to non-radiative transfer [42]. In the case of QDs [42], FRET has been observed both in solution and in solid state assembly, due to the dispersion in size of the NPs, inherent to the synthetic process [43]. Here, due to the low but not negligible

Table 1 Average decay time $\tau_{\text {AV }}$ of as-synthetized QD1 and QD2 in hexane solution and after functionalization with octanethiol (oT), propanethiol (pT) and the corresponding octanedithiol (oDT) and propanedithiol (pDT) bifunctional ligands, at the same molar ratio

\begin{tabular}{cccccc}
\hline$\tau_{\mathrm{AV}}(\mathrm{ns})$ & BUA-QD & oT QD & pT QD & oDT QD & pDT QD \\
\hline QD1 & $25.2 \pm 0.12$ & $25.1 \pm 0.41$ & $23.6 \pm 0.45$ & $16.0 \pm 0.30$ & $10.0 \pm 0.26$ \\
QD2 & $27.69 \pm 0.13$ & $27.81 \pm 0.37$ & $28.01 \pm 0.45$ & $14.62 \pm 0.40$ & $8.59 \pm 0.24$ \\
\hline
\end{tabular}


dispersion in size of the prepared QD1 and QD2, namely $\sigma \%=$ $7 \%$ and $8 \%$, respectively, the dimers, in each sample, are likely to be formed of QDs of slightly different size.

Therefore, a FRET mechanism can be depicted as the result of the interaction within a dimer between a smaller size QD, acting as a donor D, interacting with a larger QD, behaving as an acceptor A. In presence of dithiol, the observed red shift of the PL signal (black line in Fig. 4(b)) may be, in the first instance, attributed to a faint decrease of the PL intensity signal on the high energy side, due to the quenching of the donor QD and an emission increase on the low energy side due to the PL enhancement of the acceptor QD, as expected in FRET process.

However, since here D and A correspond to the same emitter, their emission and recombination decay profiles cannot be investigated separately.

In case of long chain hdDT, red-shift of the PL and asymmetric broadening of the emission curve on the low energy side are evident (Fig. S9 in the ESM). In addition, the decay in correspondence of the low and high energy side on the tails of the PL curve, for both the native QD and the QDs linked with hdDT, are compared. While the single QDs dispersed in solution behave as non-interacting units and their decay profiles at low and high energy overlap, when linked with hdDT, the emission on the high energy side decays faster with respect to the low energy side where, the decay slows down.

FRET theory [44] allows us to estimate the efficiency of the process, $E_{\text {FRET }}$, the D-A separation where energy transfer has a $50 \%$ probability of occurring, $R_{0}$, and the physical distance center to center of D-A QD, $r$, according to the equations reported in the ESM. $E_{\mathrm{FRET}}$ is determined by the ratio of average decay time $\tau_{\mathrm{DA}}$ of the QD D in the presence of the QD A over the decay time $\tau_{\mathrm{D}}$ of the single QD D in absence of the A (Eq. (S1) in the ESM). For this reason, we reasonably consider $\tau_{\mathrm{DA}}$ the lifetime of dimers in solution formed by QDs linked by means of a dithiol.

From the Eq. (S3) in the ESM and from the experimentally obtained values of $E_{\mathrm{FRET}}$ and $R_{0}$, the theoretical interparticle distance $r_{\text {theory }}$ can be calculated and the values are reported in Table 2 .

However, for dimers formed by using pDT, the $r_{\text {theory }}$ does not fit the $r_{\text {geom }}$ values, resulting, in fact, smaller than the values predicted by the Forster theory.

In Fig. 5 the FRET efficiency obtained from Eq. (S3) in the ESM for QD1 and QD2 dimers prepared with pDT and hdDT (pDT QD1, pDT QD2, hdDT QD2) respectively. $E_{\mathrm{FRET}}$ versus the center-to-center D-A separation is plotted.

The experimental points (red and black dot, respectively), corresponding to the ERRET values calculated considering the $r_{\text {geom }}$, for $\mathrm{pDT}$, deviate from the position on the corresponding theoretical curves. Such a divergence allows us to rule out dipole induced energy transfer as responsible of the observed spectroscopic effects in case of dimers with pDT as linker. Instead, for QD dimers prepared by using a long alkyl chain bifunctional ligand as hdDT, with a nominal chain length of $2.2 \mathrm{~nm}$, the

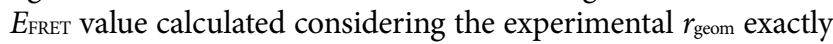
corresponds to the value on the theoretical curve (green line and dot in Fig. 5), thus confirming that a FRET process occurs between the QDs when the alkyl chain is few nm long. Therefore,

Table 2 Experimental and theoretical values of FRET parameters for pDT dimers based on QD1 and QD2 samples

\begin{tabular}{ccccccc}
\hline Sample & $\begin{array}{c}\tau_{\mathrm{D}} \\
(\mathrm{ns})\end{array}$ & $\begin{array}{c}\tau_{\mathrm{D}-\mathrm{A}} \\
(\mathrm{ns})\end{array}$ & $E_{\mathrm{FRET}}$ & $\begin{array}{c}R_{0} \\
(\mathrm{~nm})\end{array}$ & $\begin{array}{c}r_{\text {theory }} \\
(\mathrm{nm})\end{array}$ & $\begin{array}{c}r_{\text {geom }} \\
(\mathrm{nm})\end{array}$ \\
\hline pDT QD1 & 25.25 & 10 & 0.61 & 7.7 & 7.19 & 4.95 \\
pDT QD2 & 27.69 & 8.59 & 0.69 & 6.9 & 6.07 & 4.25 \\
\hline
\end{tabular}

a variation of the interparticle distance from 0.55 to $2.2 \mathrm{~nm}$ for dimers prepared by using $\mathrm{pDT}$ and hdDT, respectively, turns into a dramatic difference in the coupling mechanism.

In order to better clarify the mechanism of excitonic dissociation at a short interparticle distance, the transfer rates for QD1 and QD2 dimers prepared with the different alkyl dithiol have been calculated from the measured exciton lifetimes (Tables 1 and 2) via the equation $k_{\text {et }}=1 / \tau_{\text {(coupled) }}-1 / \tau_{\text {(isolated). }}$. The $k_{\text {et }}$ value are plotted as a function of $r_{\text {geom }}$ and reported in Fig. 6.

The calculations indicate that, firstly, decreasing the alkyl chain length, moving from oDT to $\mathrm{pDT}$, which affects directly the interparticle distance between QDs, the transfer rate increases exponentially, thus highlighting that small variation in the QD interparticle distance strongly affects the coupling efficiency. The trend (red and green line in Fig. 6) can be explained considering the Marcus theory [45] that describes the charge tunneling through a potential barrier, as also reported for PbS QD assemblies [46]. However, in contrast to the typical charge transfer occurring among nanoparticles deposited on a substrate, our assemblies are composed of two or more QDs, mainly capped with long chain insulating molecules, dispersed in an isolated environment (hexane solution), in very close

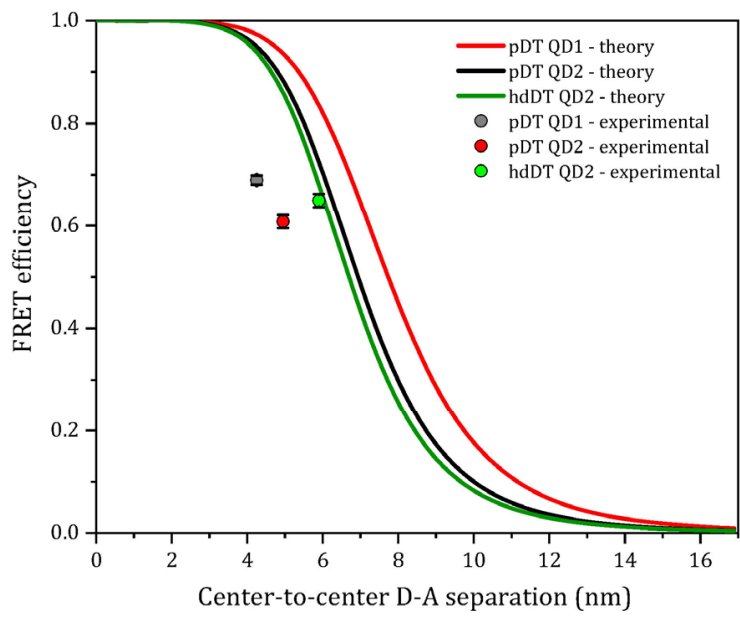

Figure 5 Theoretical FRET efficiency calculated as a function of the D-A distance for dimers prepared by using QD1, QD2 and pDT (black and red line, respectively) or hdDT (green line) as bifunctional linkers. The three dots indicate the experimental $E_{\mathrm{FRET}}$ values for the calculated geometrical center-to-center D-A distance $r_{\text {geom }}$ (given by the sum of QD diameter and alkyl chain length). The error bars are reported.

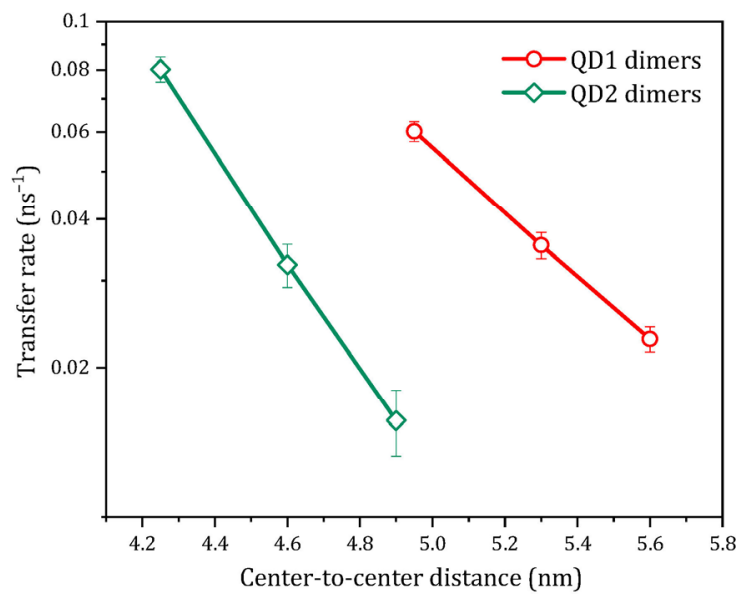

Figure 6 Plot of charge transfer rates, in logarithmic scale, calculated from exciton lifetimes, versus $r_{\text {geom }}$ for both the series of dimers fabricated starting from QD1 (red line) and QD2 (green line). The error bars are reported. 
proximity (at $0.5 \mathrm{~nm}$ of distance when pDT is used). Then, when the nanoparticles are optically excited at low intensity, a delocalization of the excitonic wavefunctions of both the QDs, overlapping in the space in between, is expected instead of a complete charge transfer between the two nanoparticles.

In addition, the transfer rate, $k_{\mathrm{et}}$, results strongly dependent from the size of the QDs forming the dimers (Fig. 6), being higher for smaller QDs. The delocalization of the wavefunction outside the QD, larger for smaller QDs [27], reflects a stronger coupling at the same interparticle distance.

Moreover, as expected, for larger distances, as for hDT or oDT, and, hence, lower wavefunction overlap and lower coupling efficiency, the transfer rate is less affected by the QD size.

Exciton dissociation in dimers with pDT as bifunctional linkers can be assumed to occur by tunneling between neighbor small QDs, that are tightly coupled. Interestingly, the interparticle distance results are distinctive for the two different mechanisms of interaction between the QDs forming a dimer in solution. Indeed, between the two QDs in a dimer an energy transfer occurs for nanometric interparticle distance, while a charge tunneling, due to a wavefunction delocalization, takes place for subnanometer distance.

The obtained results demonstrate that the control of the geometric parameters in dimers, QD size and interparticle distance, and, more generally, in larger assemblies can strongly influence the transfer rate and the coupling efficiency among the units and, consequently their electronic properties.

\section{Conclusions}

An original strategy for the fabrication of dimers of QDs in solution has been proposed by properly engineering the NP surface, first suitably pre-treating the pristine QDs, and then using dithiols as bifunctional ligands to connect them. Dimeric QDs assemblies with interparticle distance ranging from few nm down to sub-nm have been obtained by using alkyl dithiols with different chain length. The spectroscopic investigation of the assemblies clearly highlights that the QD coupling phenomena do depend on the interparticle distance and that QD size significantly affects the exciton dissociation efficiency. These findings demonstrate that it is possible to control the transfer rate in the dimers either tuning the QD interparticle distance or the size of the QD itself. Finally, the proposed assembling strategy can be extended to the fabrication in solution of dimers based on colloidal NCs with different composition and controlled size and interparticle distance, giving rise to a plethora of original assemblies. Such preparation approach can have a relevant impact both on the basic understanding of optical and electronic properties in molecular assemblies and for their possible technological applications in photonic or optoelectronic devices based on assembled QDs.

\section{Materials and method}

\subsection{Materials}

Cadmium oxide (CdO, 99.5\%), selenium (Se, 99.99\%), oleic acid (OLEA, 90\%), trioctylphosphinoxide (TOPO, 99\%), tributylphosphine (TBP, 99\%), hexadecylamine (HDA, 90\%), 1,3-propanedithiol (pDT, 99\%), 1,6-hexanedithiol (hDT, 96\%), 1,8-octanedithiol (oDT, 97\%), 1,16-hexadecanedithiol (hdDT, 99\%), 1-propanethiol (pT, 99\%), 1-octanethiol (oT, 99\%), butylamine (BUA, 99\%). All chemicals were used as received, without any further purification or distillation. Ethanol ( $\geq 99.8 \%$ ), and hexane (anhydrous $\geq 99 \%$ ) were used at analytical grade, unless otherwise specified, and purchased from Sigma-Aldrich.

\subsection{Synthesis of CdSe QDs}

A mixture of $0.127 \mathrm{~g}$ of $\mathrm{CdO}(1 \mathrm{mmol})$ and $1 \mathrm{~mL}$ of OLEA was heated at $90{ }^{\circ} \mathrm{C}$ in a three necked flask under vacuum to eliminate moisture and then heated up to $260{ }^{\circ} \mathrm{C}$ under nitrogen to completely decompose the $\mathrm{CdO}$ red powder into the Cd-oleate complex (pale yellow solution). The Cd-oleate mixture was then cooled down to $85{ }^{\circ} \mathrm{C}$ and kept under nitrogen. TOPO ( $9 \mathrm{~g}, 23 \mathrm{mmol}$ ) and HDA (9 g, $37 \mathrm{mmol})$ were put at $110^{\circ} \mathrm{C}$ under vacuum for $1 \mathrm{~h}$, cooled down to $85^{\circ} \mathrm{C}$ and transferred into the $\mathrm{Cd}$ oleate flask. The temperature was then increased up to $300{ }^{\circ} \mathrm{C}$ and $2 \mathrm{~mL}$ of TBP were injected at $280{ }^{\circ} \mathrm{C}$ followed by the Se precursor solution $(0.394 \mathrm{~g}$ of Se, $5 \mathrm{mmol}$, dissolved in $4.5 \mathrm{~mL}$ of TBP) injection at $295^{\circ} \mathrm{C}$ (injection temperature). Soon, after the injection, the temperature was low down up to $270{ }^{\circ} \mathrm{C}$ (growth temperature) and the flask was let at this temperature under stirring for $180 \mathrm{~s}$ (reaction time), for the synthesis of the QD1 samples. Similar synthetic protocol was used for the synthesis of the QD2 samples, prepared at smaller ( $90 \mathrm{~s}$ ) reaction time. To quench the QD growth the reaction mixture was cooled down and the QDs were finally collected by addition of ethanol and redispersed in $4 \mathrm{~mL}$ of hexane, resulting in concentration of $10^{-4} \mathrm{M}$ for QD1 and $2 \times 10^{-4} \mathrm{M}$ for QD2.

\subsection{QD dimers by direct ligand exchange reaction with alkyl dithiols or two steps functionalization with butylamine and alkyl dithiols}

QD colloidal solution was diluted in hexane at $5 \times 10^{-7} \mathrm{M}$ and stock solutions of each dithiol ( $\mathrm{pDT}, \mathrm{hDT}$, oDT and hdDT) at $3.3 \times 10^{-3} \mathrm{M}$ were prepared. Direct ligand exchange reaction experiments for dimers preparation were performed by adding the dithiol solution to the QD at different QD/DT molar ratio let them stir for $5 \mathrm{~min}$ at room temperature. UV-Vis absorbance and PL $\left(\lambda_{\mathrm{ex}}=485 \mathrm{~nm}\right)$ characterization were prepared without further dilution, while samples were diluted to 1:60 with hexane for TEM grid preparation and statistical analysis of dimer yield (DY).

Dimers preparation by a two steps approach were carried out for the QD1 sample. First, QDs $\left(5 \times 10^{-7} \mathrm{M}\right)$ were treated with a solution of BUA $\left(2.5 \times 10^{-2} \mathrm{M}\right)$ at BUA:QD molar ratio 500:1 (BUA: QD molar ratio in the range of 250:1 up to 20,000:1 were also investigated) and TEM and spectroscopic characterization of the samples were performed. Addiction of alkyl dithiol $\left(1.7 \times 10^{-4} \mathrm{M}\right)$ was further carried out, exploring the alkyldithiol:QD molar ratio range from 10:1 to 100:1, monitoring the evolution of the UV-Vis absorption, PL $\left(\lambda_{\mathrm{ex}}=\right.$ $485 \mathrm{~nm}$ ) and time resolved PL (TRPL) spectra $\left(\lambda_{\mathrm{ex}}=485 \mathrm{~nm}\right)$. Density gradient ultracentrifugation (DGU) procedures were carried out to improve the DY, by using solutions of cyclohexane and tetrachloroethylene at different relative ratios, from $90 \%$ to $30 \%$. The samples were centrifuged at $25,000 \mathrm{rpm}$ for $15 \mathrm{~min}$. Samples were diluted to 1:60 in hexane for TEM grid preparation and statistical analysis of DY.

The dimer yield in each sample has been calculated as the ratio between the number of dimers counted in each TEM micrograph (at least 15 different images were considered for each sample) over the total number of particles. In our analysis, assemblies formed of less than 6-8 QDs, for which it is still possible to consider the molecular nature of the assembly, are taken into account. In this sense, the obtained DY overcomes the value of $60 \%$. The remaining fraction, visible in the TEM 
images, are given by either single QDs or large aggregates. It is worth noting that the aggregates composed of tens or hundreds of nanoparticles were not considered in the calculation of the DY, due to the large error in the determination of the correct number of QDs, then underestimating the actual value of the DY.

\subsection{QD functionalization with alkyl thiols}

QD1 and QD2 samples $\left(5 \times 10^{-7} \mathrm{M}\right)$ were treated with alkyl thiols namely pT and oT $\left(3.3 \times 10^{-3} \mathrm{M}\right)$ in a QD:thiol molar ratio 1:50 for $5 \mathrm{~min}$ under stirring at room temperature. UV-Vis absorbance, PL $\left(\lambda_{\mathrm{ex}}=485 \mathrm{~nm}\right)$ and time resolved PL (TRPL) spectra $\left(\lambda_{\mathrm{ex}}=485 \mathrm{~nm}\right)$ were recorded without further sample dilution, while dilution at 1:60 with hexane was carried out for TEM grid preparation and analysis.

\subsection{Characterization techniques}

UV-Vis absorption spectra were recorded with a Cary 5000 (Varian) UV/Vis/NIR spectrophotometer.

All fluorescence measurements were performed at room temperature. Fluorescence spectra were recorded by using a Fluorolog 3 spectrofluorimeter (HORIBA Jobin-Yvon), equipped with double grating excitation and emission monochromators. Time resolved photoluminescence (TRPL) measurements were performed by Time-Correlated Single Photon Counting (TCSPC) technique, with a FluoroHub (HORIBA Jobin-Yvon). The samples were excited at $485 \mathrm{~nm}$ by means of a picosecond laser diode (NanoLED 485L) with a pulse length of 80 ps at a $1 \mathrm{MHz}$ repetition rate, with a typical power below $10 \mathrm{pJ} /$ pulse. The PL signals were dispersed by a double grating monochromator and detected by a picosecond photon counter (TBX ps Photon Detection Module, HORIBA Jobin-Yvon). The temporal resolution of the experimental set up was $\sim 200$ ps. DAS Analysis ${ }^{\circledR}$ software by Horiba was used to fit the decay profile with a multiexponential (3-4 orders) and average lifetimes were calculated.

Absolute quantum yield measurements were obtained by means of a "Quanta-phi" integrating sphere coated with Spectralons ${ }^{\circledR}$ and mounted in the optical path of the spectrofluorometer, using as an excitation source a $450 \mathrm{~W}$ xenon lamp coupled with a double-grating monochromator.

For TEM analysis, samples were prepared by dipping a carbon-coated copper grid into the QDs solution. A JEOL JEM1011 microscope, operating at an accelerating voltage of $100 \mathrm{kV}$ and equipped with a W electron source and a CCD high resolution camera was used for images acquisition. Statistical analysis of the size (QD average size and size distribution) of the samples was performed by using of a freeware image analysis program. The percentage relative standard deviation $(\sigma \%)$ was calculated for each sample, providing information on the QD size distribution. Its value is based on the distribution of size compared to the average value and is expressed as a percentage. The HRTEM images in the ESM were acquired with a JEM2200FS (JEOL)TEM (Schottky emitter), which operates at $200 \mathrm{kV}$. The High-resolution HAADF STEM images were obtained on Thermo-Fisher Scientific probe corrected Themis Z, which operates at $300 \mathrm{kV}$.

\section{Acknowledgements}

This work is financially supported by the H2020 FET project COPAC (Contract agreement n.766563).

The MIUR PRIN 2015 n. 2015XBZ5YA is also acknowledged.

Electronic Supplementary Material: Supplementary material (additional TEM, HRTEM, spectroscopic characterization, time resolved spectroscopy data, FTIR spectra and equations used for FRET calculation) is available in the online version of this article at https://doi.org/10.1007/s12274-020-2747-3.

Open Access This article is licensed under a Creative Commons Attribution 4.0 International License, which permits use, sharing, adaptation, distribution and reproduction in any medium or format, as long as you give appropriate credit to the original author(s) and the source, provide a link to the Creative Commons licence, and indicate if changes were made.

The images or other third party material in this article are included in the article's Creative Commons licence, unless indicated otherwise in a credit line to the material. If material is not included in the article's Creative Commons licence and your intended use is not permitted by statutory regulation or exceeds the permitted use, you will need to obtain permission directly from the copyright holder.

To view a copy of this licence, visit http://creativecommons.org/licenses/by/4.0/.

\section{References}

[1] Suárez Alvarez, I. Active photonic devices based on colloidal semiconductor nanocrystals and organometallic halide perovskites. Eur. Phys. J. Appl. Phys. 2016, 75, 30001.

[2] Kovalenko, M. V.; Manna, L.; Cabot, A.; Hens, Z.; Talapin, D. V.; Kagan, C. R.; Klimov, V. I.; Rogach, A. L.; Reiss, P.; Milliron, D. J. et al. Prospects of nanoscience with nanocrystals. ACS Nano 2015, 9, 1012-1057.

[3] Litvin, A. P.; Martynenko, I. V.; Purcell-Milton, F.; Baranov, A. V.; Fedorov, A. V.; Gun'ko, Y. K. Colloidal quantum dots for optoelectronics. J.Mater. Chem. A2017, 5, 13252-13275.

[4] Kim, T. H.; Cho, K. S.; Lee, E. K.; Lee, S. J.; Chae, J.; Kim, J. W.; Kim, D. H.; Kwon, J. Y.; Amaratunga, G.; Lee, S. Y. et al. Fullcolour quantum dot displays fabricated by transfer printing. Nat. Photonics 2011, 5, 176-182.

[5] Shirasaki, Y.; Supran, G. J.; Bawendi, M. G.; Bulović, V. Emergence of colloidal quantum-dot light-emitting technologies. Nat. Photonics 2013, 7, 13-23.

[6] Zhao, T. S.; Goodwin, E. D.; Guo, J. C.; Wang, H.; Diroll, B. T.; Murray, C. B.; Kagan, C. R. Advanced architecture for colloidal PbS quantum dot solar cells exploiting a CdSe quantum dot buffer layer. ACS Nano 2016, 10, 9267-9273.

[7] Carey, G. H.; Abdelhady, A. L.; Ning, Z. J.; Thon, S. M.; Bakr, O. M.; Sargent, E. H. Colloidal quantum dot solar cells. Chem. Rev. 2015, 115, 12732-12763.

[8] Morales-Narváez, E.; Golmohammadi, H.; Naghdi, T.; Yousefi, H.; Kostiv, U.; Horák, D.; Pourreza, N.; Merkoçi, A. Nanopaper as an optical sensing platform. ACS Nano 2015, 9, 7296-7305.

[9] Wang, G.; Leng, Y. K.; Dou, H. J.; Wang, L.; Li, W. W.; Wang, X. B.; Sun, K.; Shen, L. S.; Yuan, X. L.; Li, J. Y.et al. Highly efficient preparation of multiscaled quantum dot barcodes for multiplexed hepatitis B detection. ACS Nano 2013, 7, 471-481.

[10] Lee, J. S.; Kovalenko, M. V.; Huang, J.; Chung, D. S.; Talapin, D. V. Band-like transport, high electron mobility and high photoconductivity in all-inorganic nanocrystal arrays. Nat. Nanotechnol. 2011, 6, $348-352$.

[11] Semonin, O. E.; Luther, J. M.; Beard, M. C. Quantum dots for nextgeneration photovoltaics. Mater. Today 2012, 15, 508-515.

[12] Balazs, D. M.; Rizkia, N.; Fang, H. H.; Dirin, D. N.; Momand, J.; Kooi, B. J.; Kovalenko, M. V.; Loi, M. A. Colloidal quantum dot inks for single-step-fabricated field-effect transistors: The importance of postdeposition ligand removal. ACS Appl. Mater. Interfaces 2018, $10,5626-5632$.

[13] Zhang, H. T.; Hu, B.; Sun, L. F.; Hovden, R.; Wise, F. W.; Muller, D. A.; Robinson, R. D. Surfactant ligand removal and rational fabrication of inorganically connected quantum dots. Nano Lett. 2011, 11, 5356-5361.

[14] Cohen, E.; Komm, P.; Rosenthal-Strauss, N.; Dehnel, J.; Lifshitz, E.; Yochelis, S.; Levine, R. D.; Remacle, F.; Fresch, B.; Marcus, G. 
et al. Fast energy transfer in CdSe quantum dot layered structures: Controlling coupling with covalent-bond organic linkers. J.Phys. Chem. C 2018, 122, 5753-5758.

[15] Talapin, D. V.; Murray, C. B. PbSe nanocrystal solids for $\mathrm{n}-$ and p-channel thin film field-effect transistors. Science 2005, 310, 86-89.

[16] Zhao, K.; Mason, T. G. Assembly of colloidal particles in solution. Rep. Prog. Phys. 2018, 81, 126601.

[17] Romo-Herrera, J. M.; Alvarez-Puebla, R. A.; Liz-Marzán, L. M. Controlled assembly of plasmonic colloidal nanoparticle clusters. Nanoscale 2011, 3, 1304-1315.

[18] Yu, L.; Shiraishi, S.; Wang, G. Q.; Akiyama, Y.; Takarada, T.; Maeda, M. Connecting nanoparticles with different colloidal stability by DNA for programmed anisotropic self-assembly. J.Phys. Chem. C 2019, 123, 15293-15300.

[19] Wang, X. J.; Li, G. P.; Chen, T.; Yang, M. X.; Zhang, Z.; Wu, T.; Chen, H. Y. Polymer-encapsulated gold-nanoparticle dimers: Facile preparation and catalytical application in guided growth of dimeric ZnO-nanowires. Nano Lett. 2008, 8, 2643-2647.

[20] Chen, G.; Wang, Y.; Tan, L. H.; Yang, M. X.; Tan, L. S.; Chen, Y.; Chen, H. Y. High-purity separation of gold nanoparticle dimers and trimers. J. Am. Chem. Soc. 2009, 131, 4218-4219.

[21] Zohar, N.; Chuntonov, L.; Haran, G. The simplest plasmonic molecules: Metal nanoparticle dimers and trimers. J. Photochem. Photobiol. C 2014, 21, 26-39.

[22] Fernandez, Y. D.; Sun, L. L.; Gschneidtner, T.; Moth-Poulsen, K. Research update: Progress in synthesis of nanoparticle dimers by self-assembly. APL Mater. 2014, 2, 010702.

[23] Yamashita, N.; Ma, Z. P.; Park, S.; Kawai, K.; Hirai, Y.; Tsuchiya, T.; Tabata, O. Formation of gold nanoparticle dimers on silicon by sacrificial DNA origami technique. Micro Nano Lett. 2017, 12, 854-859.

[24] Thacker, V. V.; Herrmann, L. O.; Sigle, D. O.; Zhang, T.; Liedl, T.; Baumberg, J. J.; Keyser, U. F. DNA origami based assembly of gold nanoparticle dimers for surface-enhanced Raman scattering. Nat. Commun. 2014, 5, 3448.

[25] Hanrath, T. Colloidal nanocrystal quantum dot assemblies as artificial solids. J. Vac. Sci.Technol. A 2012, 30, 030802.

[26] Peng, X.G.; Wilson, T. E.; Alivisatos, A. P.; Schultz, P. G. Synthesis and isolatin of a homodimer of cadmium selenide nanocrystals. Angew. Chem., Int. Ed. 1997, 36, 145-147.

[27] Koole, R.; Liljeroth, P.; de Mello Donegá, C.; Vanmaekelbergh, D.; Meijerink, A. Electronic coupling and exciton energy transfer in CdTe quantum-dot molecules. J. Am. Chem. Soc. 2006, 128, 10436-10441.

[28] Xu, X.X.; Stöttinger, S.; Battagliarin, G.; Hinze, G.; Mugnaioli, E.; Li, C.; Müllen, K.; Basché, T. Assembly and separation of semiconductor quantum dot dimers and trimers. J. Am. Chem. Soc. 2011, 133, 18062-18065.

[29] Tanford, C. The Hydrophobic Effect: Formation of Micelles and Biological Membranes; 2nd ed. John Wiley\& Sons: New York, 1980.

[30] Wu, S. S.; McGuigan, M.; Tiano, A. L.; Wong, S. S.; Glimm, J. G. A first-principles study of CdSe NANOCLUSTERS capped by thiol ligands. arXiv:1308.4671, 2013.

[31] Altomare, M.; Fanizza, E.; Corricelli, M.; Comparelli, R.; Striccoli,
M.; Curri, M. L. Patterned assembly of luminescent nanocrystals: Role of the molecular chemistry at the interface. J. Nanopart. Res. 2014, 16, 2468.

[32] Anderson, N. C.; Hendricks, M. P.; Choi, J. J.; Owen, J. S. Ligand exchange and the stoichiometry of metal chalcogenide nanocrystals: Spectroscopic observation of facile metal-carboxylate displacement and binding. J. Am. Chem. Soc. 2013, 135, 18536-18548.

[33] Kumar, A. P.; Huy, B. T.; Kumar, B. P.; Kim, J. H.; Dao, V. D.; Choi, H. S.; Lee, Y. I. Novel dithiols as capping ligands for CdSe quantum dots: Optical properties and solar cell applications. J. Mater. Chem. C2015, 3, 1957-1964.

[34] Hostetler, M. J.; Templeton, A. C.; Murray, R. W. Dynamics of placeexchange reactions on monolayer-protected gold cluster molecules. Langmuir 1999, 15, 3782-3789.

[35] McCarthy, C. L.; Brutchey, R. L. Solution processing of chalcogenide materials using thiol-amine "alkahest" solvent systems. Chem. Commun. 2017, 53, 4888-4902.

[36] Mei, B. C.; Oh, E.; Susumu, K.; Farrell, D.; Mountziaris, T. J.; Mattoussi, H. Effects of ligand coordination number and surface curvature on the stability of gold nanoparticles in aqueous solutions. Langmuir 2009, 25, 10604-10611.

[37] Talapin, D. V.; Rogach, A. L.; Kornowski, A.; Haase, M.; Weller, H. Highly luminescent monodisperse CdSe and CdSe/ZnS nanocrystals synthesized in a hexadecylamine-trioctylphosphine oxidetrioctylphospine mixture. Nano Lett. 2001, 1, 207-211.

[38] Michen, B.; Geers, C.; Vanhecke, D.; Endes, C.; Rothen-Rutishauser, B.; Balog, S.; Petri-Fink, A. Avoiding drying-artifacts in transmission electron microscopy: Characterizing the size and colloidal state of nanoparticles. Sci. Rep. 2015, 5, 9793.

[39] Wuister, S. F.; de Mello Donegá, C.; Meijerink, A. Influence of thiol capping on the exciton luminescence and decay kinetics of CdTe and CdSe quantum dots. J.Phys. Chem. B 2004, 108, 17393-17397.

[40] Piston, D. W.; Kremers, G. J. Fluorescent protein FRET: The good, the bad and the ugly. Trends Biochem. Sci. 2007, 32, 407-414.

[41] Sapsford, K. E.; Berti, L.; Medintz, I. L. Materials for fluorescence resonance energy transfer analysis: beyond traditional donor-acceptor combinations. Angew. Chem., Int. Ed. 2006, 45, 4562-4589.

[42] Chou, K. F.; Dennis, A. M. Förster resonance energy transfer between quantum dot donors and quantum dot acceptors. Sensors 2015, 15, 13288-13325.

[43] De Luca, A.; Depalo, N.; Fanizza, E.; Striccoli, M.; Curri, M. L.; Infusino, M.; Rashed, A. R.; La Deda, M.; Strangi, G. Plasmon mediated super-absorber flexible nanocomposites for metamaterials. Nanoscale 2013, 5, 6097-6105.

[44] Lakowicz, J. R.Principles of Fluorescence Spectroscopy. Springer: New York, 2006; pp 443-475.

[45] Marcus, R. A.; Sutin, N. Electron transfers in chemistry and biology. Biochim.Biophys. Acta 1985, 811, 265-322.

[46] Choi, J. J.; Luria, J.; Hyun, B. R.; Bartnik, A. C.; Sun, L. F.; Lim, Y. F.; Marohn, J. A.; Wise, F. W.; Hanrath, T. Photogenerated exciton dissociation in highly coupled lead salt nanocrystal assemblies. Nano Lett. 2010, 10, 1805-1811. 